\title{
Enhancing Network Output Load in IEEE 802.15.4 with Different Modulations for Wireless Sensor Networks
}

\author{
Sukhvinder S Bamber \\ Department of Computer Science \& Engg. \\ National Institute of Technology \\ Jalandhar, Punjab, India
}

\author{
Ajay K Sharma \\ Department of Computer Science \& Engg. \\ National Institute of Technology \\ Jalandhar, Punjab, India
}

\begin{abstract}
This paper presents the network output load at the Fully Functional Device (FFD) and Reduced Functional Devices (RFD's) of IEEE 802.15.4 for different modulation schemes. From the simulations it is revealed that Minimum Shift Keying (MSK) is best suited for all types of devices in 802.15.4 for wireless sensor networks (WSNs) if the network output load is to be maximized as more is the output load, more will be the net throughput. Simulations also reveal that Binary Phase Shift keying (BPSK) at all type of devices and Quadrature Amplitude Modulation of 64 bits (QAM_64) at the PAN (Personal Area Network) coordinator are unsuitable.
\end{abstract}

\section{Keywords}

Network output load, RFD, FFD, BPSK, MSK, QAM_64.

\section{INTRODUCTION}

In the past decade several short range wireless technologies have been developed as an answer to the increasing demand for portable and flexible connectivity. In addition to the upsurge in the deployment of IEEE 802.11 based Wireless Local Area Networks (WLANs), few complementary low-power and lowcost technologies, among which IEEE 802.15.4, are establishing their place on the market as enablers of the emerging Wireless Sensor Networks (WSNs). The IEEE 802.15.4 standard was specifically developed to address a demand for low-power, lowbit rate connectivity towards small and embedded devices. Furthermore the standard is trying to solve some problems that were inadequately taken into account by Bluetooth technology.

The choice of the digital modulation scheme in IEEE 802.15.4 significantly affects the output load at the different types of devices in wireless sensor communication system. There is no universal 'best' choice of the modulation scheme, but depending on the physical characteristics of the channel, parametric optimizations and required level of performance some will prove better fit than the others. The 802.15.4 is an IEEE standard, targeting a set of applications that require simple wireless connectivity, high throughput, very low power consumption and lower module cost.

Despite the intense research and standardization tasks performed over the last years on maximizing the network output load, still many open issues are to be dealt with before wireless sensor networks can be utilized on large scale. Researchers have carried out the research in various areas [1-12]. Some have investigated various performance issues like: Delay, Throughput evaluation of GTS mechanism [1]. Few have studied load balancing in wireless mesh networks [4]. Some researchers have tried to distribute the load in wireless sensor networks [6]. Few others have forecasted peak load in the neural networks [10]. Some others have coupled the network load for different applications [12].

But none of the researchers have so far compared the modulation schemes for maximizing the network output load at different types of devices in IEEE 802.15.4 for wireless sensor networks. This paper proposes the comparison of three different modulation schemes (MSK, BPSK, QAM_64) to determine the suitability of the scheme according to the device type (FFD/RFD) to maximize the network output load.

The paper is outlined as: Section [1] consists of the Introduction. Section [2] constitutes the system description which contains node model, process model, and parametric tables of the model. Section [3] shows the results and discussions derived from the experiments carried out on 802.15.4 for different modulation schemes. Finally Section [4] concludes the paper.

\section{SYSTEM DESCRIPTION}

The simulation model implements physical and medium access layers defined in IEEE 802.15.4 standard. The OPNET® Modeler 14.5 is used for developing 802.15.4 wireless sensor network.

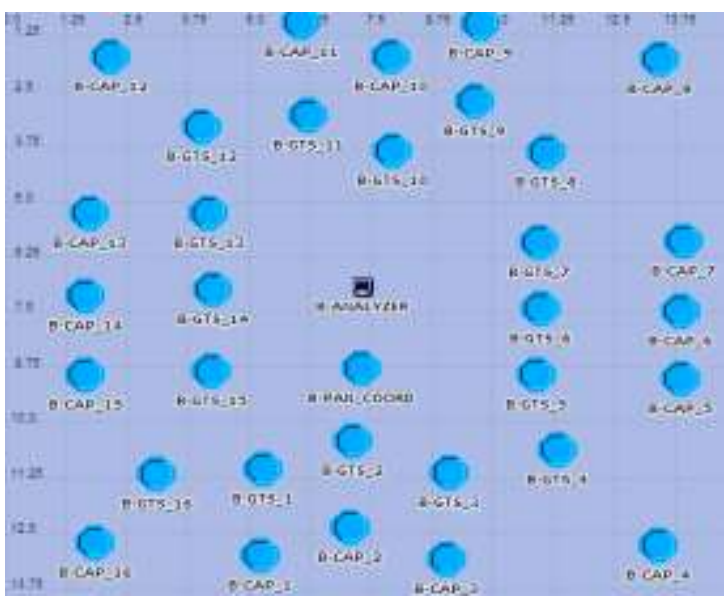

(a) 


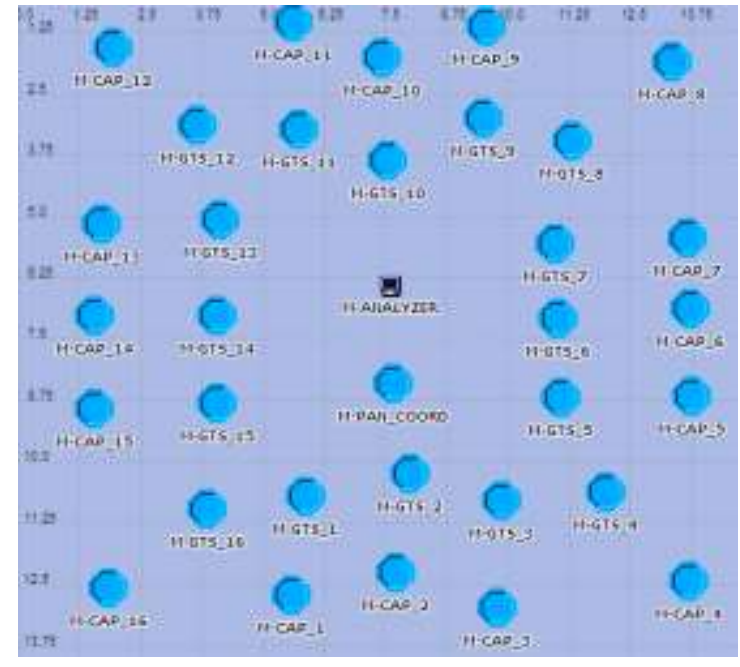

(b)

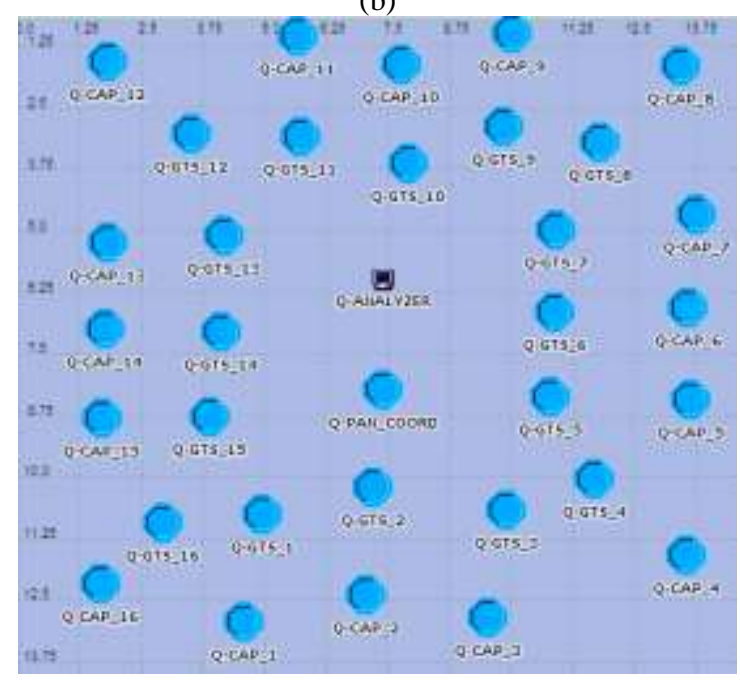

(c)

Figure 1. Network Scenarios (a) BPSK (b) MSK (c) Quadrature (QAM_64)

Figure 1 shows three different Scenarios: BPSK, MSK and QAM_64. BPSK Scenario as shown in Figure 1(a) contains one PAN Coordinator, one analyzer and thirty two end devices out of which sixteen are Guaranteed Time Slots (GTS) enabled and rest are non GTS devices. PAN Coordinator is a fully functional device which manages whole functioning of the network. Analyzer is a routing device which routes the data between PAN coordinator and the End Devices. End Devices are the fixed stations that communicate with the PAN Coordinator in Peer to Peer mode, support GTS and non GTS traffic respectively. Similar Scenarios have been created for MSK and QAM_64 as shown in figure 1 (b \& c).

Figure 2 shows the node models for three types of Wireless Personal Area Network (WPAN) devices used for modeling 802.15.4 scenarios. PAN Coordinator, GTS and Non GTS end device have the same node model as shown in Figure 2 (a) while the node model for analyzer is depicted in Figure 2 (b).

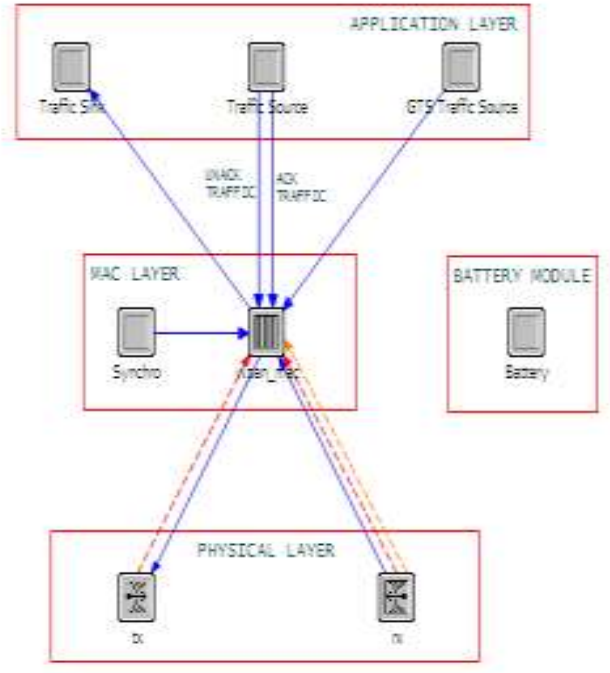

(a)

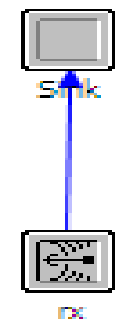

(b)

Figure 2. Node Model (a) PAN Coordinator, GTS and Non GTS end device (b) Analyzer

As it has been observed from the Figure 2 (a), a node model for PAN Coordinator, GTS end device and Non GTS end device has three layers: physical, MAC and application layers. Physical layer consists of a transmitter and a receiver compliant to the IEEE 802.15.4 specification, operating at $2.4 \mathrm{GHz}$ frequency band and data rate equal to $250 \mathrm{kbps}$. MAC layer implements slotted CSMA/CA and GTS mechanisms. The GTS data traffic coming from the application layer is stored in a buffer with a specified capacity and dispatched to the network when the corresponding GTS is active. The non time-critical data frames are stored in an unbounded buffer and based on slotted CSMA/CA algorithm are transmitted to the network during the active Contention Access Period (CAP). This layer is also responsible for the generation of beacon frames and synchronizing the network when a given node acts as a PAN Coordinator. Finally is the topmost application layer which is responsible for generation and reception of traffic consists of two data traffic generators (i.e. Traffic Source and GTS Traffic Source) and one traffic sink. The traffic source generates acknowledged and unacknowledged data frames transmitted 
during CAP. GTS traffic source can produce acknowledged and unacknowledged time-critical data frames using GTS mechanism. The traffic sink module receives frames forwarded from lower layers. Figure 2 (b) shows the node model for the analyzer which consists of sink and a radio receiver.

Corresponding process models for PAN Coordinator, GTS end device, Non GTS end device and analyzer that deals with each and every operation on the data are depicted in Figure 3:

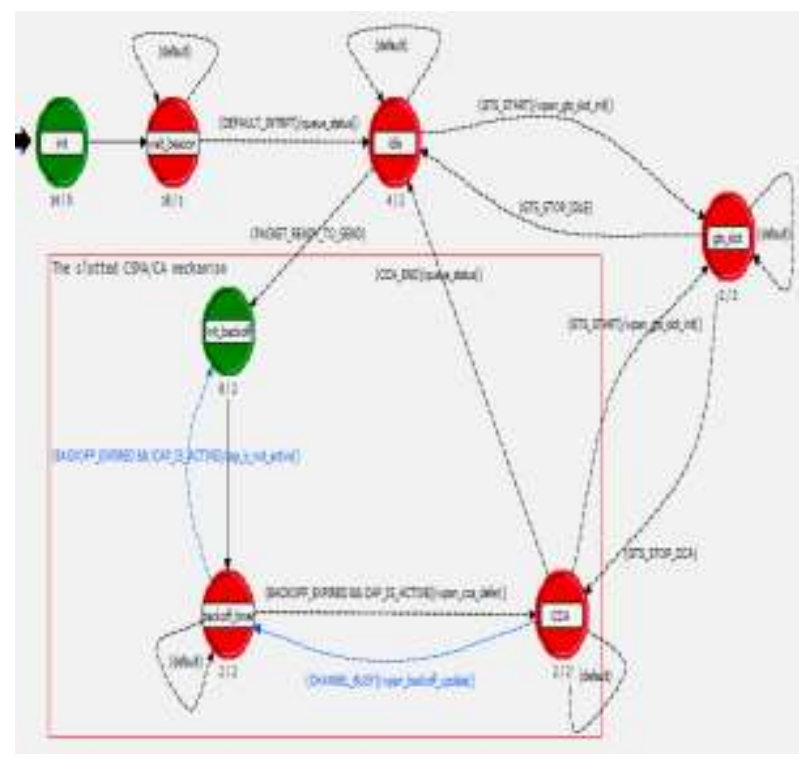

(a)

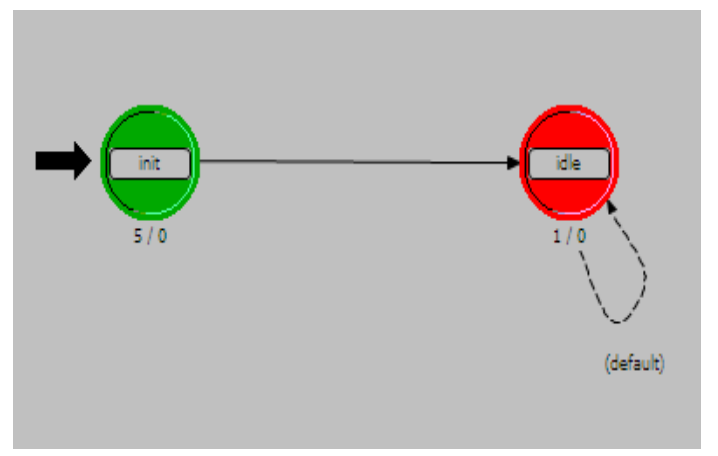

(b)

Figure 3. Process model (a) PAN Coordinator, GTS and Non GTS end device (b) Analyzer

Figure 3 (a) shows the process model for the PAN Coordinator, GTS and Non GTS end device. It consists of the various states: Init whose function is to initialize MAC and GTS scheduling; Wait_beacon which is responsible for synchronizing the traffic of the node with rest of the WPAN in order to minimize the collisions; Idle which is responsible for introducing delays in order to make the maximum use of the resources; gts_slot which is responsible for generation, reception and management of GTS traffic; Backoff_timer used for sensing the medium and transfer of data, CCA - for interrupt processing. Similarly figure 3 (b) shows the process model for analyzer which consists of init and idle states. Basically the process model explains how the data is sent from the generating node to the PAN Coordinator, taking into consideration the availability of PAN Coordinator as it has to communicate with the other similar nodes.

Here three different Scenarios have been created with three different modulation formats: BPSK, MSK and QAM_64. Following parameters have been set for these scenarios as shown in the table 1 like: destination MAC address (Acknowledged Traffic Source) of the PAN coordinator is broadcast while for GTS enabled end device and Non GTS end device the value is common i.e. PAN coordinator. Similarly the value of Superframe order in WPAN Settings is 6 (common for PAN coordinator, GTS end device and Non GTS end device).

\section{Table 1}

Parametric values for PAN Coordinator, GTS and Non GTS End Device in BPSK, MSK and QAM_64 Scenarios

\begin{tabular}{|c|c|c|c|}
\hline $\begin{array}{l}\text { Parameter } \backslash \\
\text { Scenario }\end{array}$ & $\begin{array}{c}\text { PAN } \\
\text { Coordinator }\end{array}$ & $\begin{array}{c}\text { GTS } \\
\text { Enabled } \\
\text { End } \\
\text { Device }\end{array}$ & $\begin{array}{c}\text { Non GTS } \\
\text { End Device }\end{array}$ \\
\hline Modulation & \multicolumn{3}{|c|}{ BPSK, MSK, QAM_64 } \\
\hline \multicolumn{4}{|c|}{ Acknowledged Traffic Source } \\
\hline $\begin{array}{c}\text { Destination MAC } \\
\text { Address }\end{array}$ & Broadcast & \multicolumn{2}{|c|}{ PAN Coordinator } \\
\hline $\begin{array}{c}\text { MSDU } \\
\text { Interarrival Time } \\
(\mathrm{sec})\end{array}$ & Exponential(1.0) & $\begin{array}{l}\text { Constant } \\
\text { (1.0) }\end{array}$ & Exponential(1.0) \\
\hline MSDU Size (bits) & Exponential(912) & $\begin{array}{l}\text { Constant } \\
(0.0)\end{array}$ & Exponential(912) \\
\hline Start Time (sec) & 0.0 & Infinity & 1.0 \\
\hline Stop Time (sec) & \multicolumn{3}{|c|}{ Infinity } \\
\hline \multicolumn{4}{|c|}{ Unacknowledged Traffic Source } \\
\hline $\begin{array}{c}\text { MSDU } \\
\text { Interarrival Time } \\
(\mathrm{sec})\end{array}$ & Exponential(1.0) & $\begin{array}{c}\text { Constant } \\
\text { (1.0) }\end{array}$ & Exponential(1.0) \\
\hline MSDU Size (bits) & Exponential(912) & $\begin{array}{l}\text { Constant } \\
(0.0)\end{array}$ & Exponential(912) \\
\hline Start Time (sec) & 0.1 & Infinity & 1.1 \\
\hline Stop Time (sec) & \multicolumn{3}{|c|}{ Infinity } \\
\hline \multicolumn{4}{|c|}{ CSMA/CA Parameters } \\
\hline $\begin{array}{l}\text { Maximum Back- } \\
\text { off Number }\end{array}$ & \multicolumn{3}{|c|}{4} \\
\hline $\begin{array}{l}\text { Minimum Back- } \\
\text { off Exponent }\end{array}$ & \multicolumn{3}{|c|}{3} \\
\hline \multicolumn{4}{|c|}{ IEEE 802.15 .4} \\
\hline Device Mode & PAN coordinator & \multicolumn{2}{|c|}{ End Device } \\
\hline MAC Address & \multicolumn{3}{|c|}{ Auto Assigned } \\
\hline \multicolumn{4}{|c|}{ WPAN Settings } \\
\hline Beacon Order & 14 & & 7 \\
\hline Superframe Order & \multicolumn{3}{|c|}{6} \\
\hline PAN ID & \multicolumn{3}{|c|}{0} \\
\hline \multicolumn{4}{|c|}{ Logging } \\
\hline Enable Logging & \multicolumn{3}{|c|}{ Enabled } \\
\hline \multicolumn{4}{|c|}{ GTS Settings } \\
\hline GTS Permit & \multicolumn{3}{|c|}{ Enabled } \\
\hline
\end{tabular}




\begin{tabular}{|c|c|c|c|}
\hline Start Time & 0.0 & 0.1 & Infinity \\
\hline Stop Time & \multicolumn{2}{|c|}{ Infinity } & 0 \\
\hline Length (slots) & 1 & \multicolumn{2}{c|}{ Transmit } \\
\hline Direction & Receive & 1000 \\
\hline $\begin{array}{c}\text { Buffer Capacity } \\
\text { (bits) }\end{array}$ & 10,000 & Constant (1.0) \\
\hline \multicolumn{3}{|c|}{ GTS Traffic Parameters } \\
\hline $\begin{array}{c}\text { MSDU } \\
\text { Interarrival Time } \\
\text { (sec) }\end{array}$ & Exponential(1.0) & Constant (0.0) \\
\hline \begin{tabular}{c} 
MSDU Size (bits) \\
\hline Acknowledgement
\end{tabular} & Exponential(912) & Disabled \\
\hline
\end{tabular}

\section{RESULTS AND DISCUSSIONS}

Simulative study has been carried out for the three different scenarios of 802.15.4: Quadrature (QAM_64), MSK and BPSK. In this section simulative results for the network output load have been presented and discussed for different types of devices in 802.15.4 for wireless sensor networks like: Fully functional Devices (FFD) - those devices that communicate with each and every device in the network in point to point mode and manage the routing tables, Reduced Functional Devices (RFD) - those devices which can communicate only to the FFD but not to the other RFD's.

\subsection{Network Output Load at FFD - PAN Coordinator}

Figure 4 below indicates that the network output load at the PAN coordinator is: $41936.9 \mathrm{bits} / \mathrm{sec}$ for MSK and almost comparable for BPSK and QAM_64 which is: 33623.3 bits/sec. It is observed that network output load is maximum in case of MSK as it is spectrally efficient due to it constant envelope, good BER and self synchronizing capability. Also MSK suffers less from the adjacent signal interferences as it has smooth phase shifts at the symbol boundaries which results in the lower side lobes as compared to other modulation schemes [7, 11]. Also it is observed that load is minimum in case of BPSK \& QAM_64 (almost comparable) as BPSK is able to modulate only $1 \mathrm{bit} / \mathrm{sec}$ and QAM_64 is more susceptible to noise as the decision points are more close to each other [9].

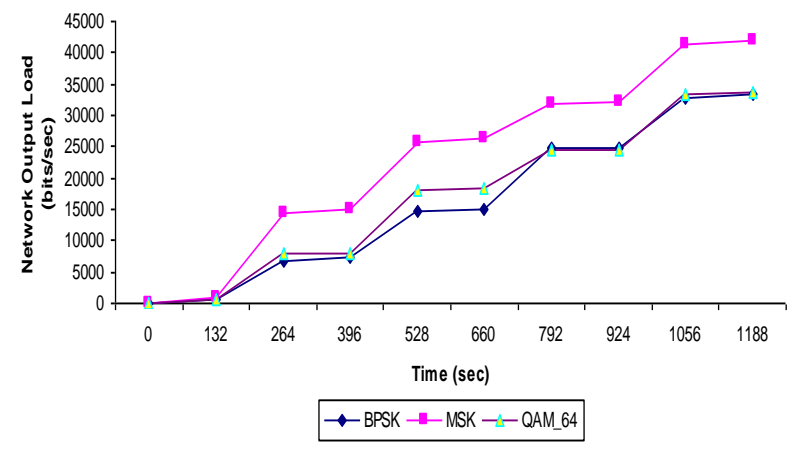

Figure 4. Network Output Load at the PAN Coordinator

\subsection{Network Output Load at RFD - GTS End Device}

Figure 5 below depicts that the network output load at the GTS end device is: 4456.6, 502.3 and $334.3 \mathrm{bits} / \mathrm{sec}$ for MSK, QAM_64 and BPSK respectively. It is observed that network output load is maximum in case of MSK [7, 11].Also it has been observed that load is minimum in case of BPSK [9].

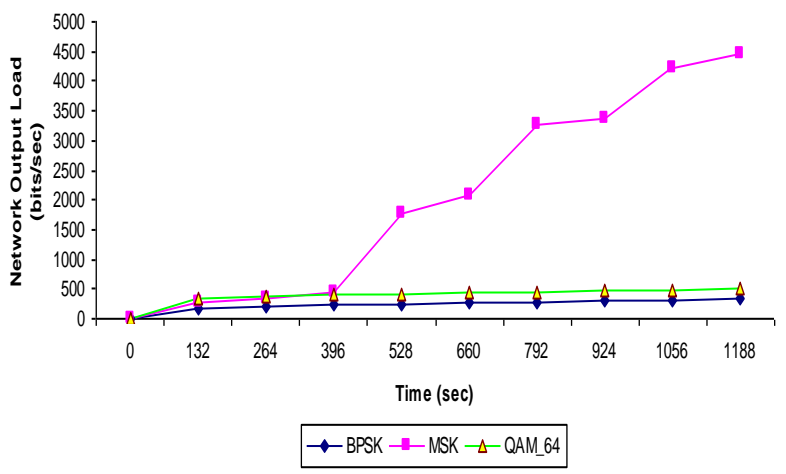

Figure 5. Network Output Load at the GTS End Device

\subsection{Network Output Load at RFD - GTS End Device}

Figure 6 below shows that the network output load at the Non GTS end device is: 40518.7, 33469.3 and $29240.8 \mathrm{bits} / \mathrm{sec}$ for MSK, QAM_64 and BPSK respectively. It is observed network load is maximum in case of MSK [7, 11]. Also it has been observed that network output load is minimum in case of BPSK [9].

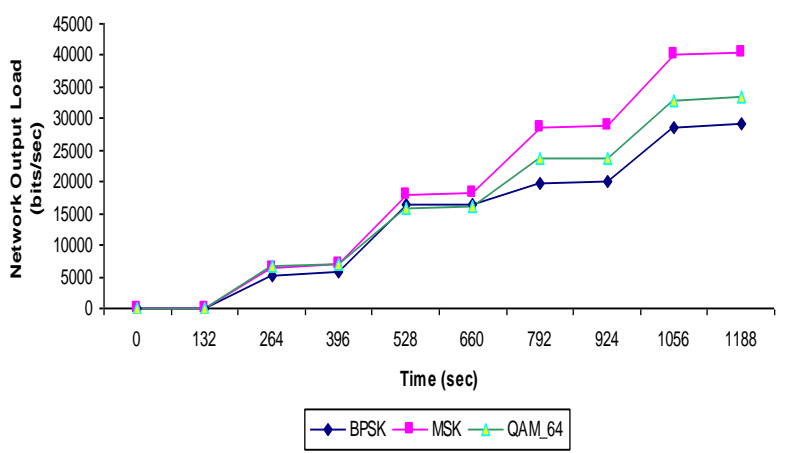

Figure 6. Network Output Load at the Non GTS End Device

\section{CONCLUSIONS}

The focus of this paper is on maximizing the network output load at different types of devices in IEEE 802.15.4 with different modulation schemes for WSNs as more is the network output load more is the net throughput. Results reveal that network load at the PAN coordinator is [41936.9, 33623.3, 33623.3] bits/sec 
for MSK, BPSK and QAM_64 respectively. Also results reveal that output load at the GTS end device and Non GTS end device is: [4456.6, 502.3, 334.3] and [40518.7, 33469.3, 29240.8] bits/sec for MSK, QAM_64 and BPSK respectively. It is concluded that if the output load in a network is to be maximized then MSK should be implemented in IEEE 802.15.4 for WSNs. Also it is concluded that BPSK at all types of devices in IEEE 802.15.4 is unsuitable for WSNs. Furthermore it is concluded that QAM_64 is also unsuitable at the PAN coordinator if IEEE 802.15.4 is to be converted into WSN.

\section{REFERENCES}

1. Jurcik, P.; Koubaa, A.; Alves, M.; Tovar, E.; Hanzalek, Z.; "A Simulation Model for the IEEE 802.15.4 protocol: Delay/Throughput Evaluation of the GTS Mechanism", Modeling, Analysis, and Simulation of Computer and Telecommunication Systems, 2007. MASCOTS '07. 15th International Symposium, 24-26 Oct. 2007.

2. IEEE 802.15.4 OPNET Simulation Model, http://www.open-zb.net/

3. Jan Magne Tjensvold, "Comparision of the IEEE $802.11,802.15 .1,802.15 .4$ and 802.15.6 wireless standards"; September 18, 2007.
4. Yigal Bejerano, Seung-Jae Han, Amit Kumar, "Efficient Load-Balancing routing for wireless mesh networks", Elsevier, COMNET, 2007.

5. Jason Lowe, "Advanced Upstream Modulation"; Clearcable Technical Summit, July 27, 2007.

6. Adel Ali Ahmed, Norsheila Fisal, "A real-time routing protocol with load distribution in wireless sensor networks", Elsevier, Computer Communications, 2008.

7. Subhash Nanjunde Gowda; "Minimum shift Keying"; Spread Spectrum Systems - 24 May 2004.

8. Emilio Ancillotti, Raffaele Bruno, Marco Conti, Antonio Pinizzotto, "Load-aware routing in mesh networks: Models, algorithms and experimentation", Elsevier, Computer Communications, 2010.

9. http://www.en.wikipedia.org.

10. P. K. Dash, A. C. Liew, S. Rahman, "Peak load forecasting using a fuzzy neural network", Elsevier, Electric Power Systems Research, 1995.

11. Charan Langton, "Intuitive Guide to Principles of Communication", www.complextoreal.com, Dec, 2005.

12. Renato Negra, Alexandre Sadeve, Souheil Bensmida, Fadhel M. Ghannouchi, "Concurrent Dual-Band Class - F Load Coupling Network for Applications at 1.7 and $2.4 \mathrm{Ghz",} \mathrm{IEEE,} 2008$. 